\title{
Symmetry and elongation of objects influence perceived direction of translational motion
}

\author{
KAZUNORI MORIKAWA \\ Rutgers University, Piscataway, New Jersey
}

\begin{abstract}
Five experiments were conducted to examine how perceived direction of motion is influenced by aspects of shape of a moving object such as symmetry and elongation. Random polygons moving obliquely were presented on a computer screen and perceived direction of motion was measured. Experiments 1 and 2 showed that a symmetric object moving off the axis of symmetry caused motion to be perceived as more aligned with the axis than it actually was. However, Experiment 3 showed that motion did not influence perceived orientation of symmetry axis. Experiment 4 revealed that symmetric shapes resulted in faster judgments on direction of motion than asymmetric shapes only when the motion is along the axis. Experiment 5 showed that elongation causes a bias in perceived direction of motion similar to effects of symmetry. Existence of such biases is consistent with the hypothesis that in the course of evolution, the visual system has been adapted to regularities of motion in the animate world.
\end{abstract}

The importance of symmetry in form perception is a long-standing principle of Gestalt psychology. Wertheimer (1958) and Hochberg (1978) proposed that symmetry is one of the determinants of perceptual organization: Symmetrical areas tend to be perceived as figure as opposed to ground. Symmetrical objects stand out more easily against their backgrounds than asymmetric objects. The axis of symmetry helps define directionality of the object (Pani, in press; Rock, 1973). In addition, symmetry improves shape recognition by establishing an object-centered coordinate frame (Barlow \& Reeves, 1979; Rock, 1973). Marr (1982) claimed that symmetry is one of the primary cues to the proper assignment of an object-centered coordinate frame onto the 21/2-D sketch of an object. McBeath, Schiano, and Tversky (1997) showed that people have a bias to interpret nearly symmetric shapes as perfectly symmetric shapes tilted in depth.

Humans seem to have very efficient symmetry-detection capabilities. Symmetry can be detected globally with only 25-msec exposure (Carmody, Nodine, \& Locher, 1977) and without conscious analysis (Barlow \& Reeves, 1979). Corballis (1976) suggested, "We may know that a shape is symmetrical before we know what else it is" (p. 77). Perceptual sensitivity to symmetry is not limited to the human visual system. In some species of birds, for example, females prefer as mates males with symmetric markings or tail feathers to males with asymmetric ones (Møller, 1992; Swaddle \& Cuthill, 1994).

Why do we possess such an efficient ability to detect symmetry? There are a few ecological advantages for visual systems that are sensitive to symmetry. Freyd and

I am grateful to Bela Julesz for his support. I also thank Thomas Papathomas and Carol Esso for their helpful comments. Correspondence should be addressed to K. Morikawa, Laboratory of Vision Research, Psychology Building, Busch Campus, Rutgers University, Piscataway, NJ 08854 (e-mail: kaz@zeus.rutgers.edu).
Tversky (1984) discussed two basic ecological values of symmetry detection: (1) Most biological and manufactured objects have at least one axis of bilateral symmetry, and thus symmetry is a useful cue for detecting and identifying objects (see also Enquist \& Arak, 1994); (2) symmetry in an image allows it to be perceived and coded abstractly and economically (see also Pani, in press).

There seems to be another advantage. It is often vital for an animal to predict the direction of motion of other animals as quickly as possible, whether the animal is fleeing from a predator, chasing prey, pouncing on a target, or throwing a spear at game. These animals are most likely symmetric objects that move along the plane of bilateral symmetry of their bodies. Therefore, being able to quickly perceive global symmetry of animate creatures could help an animal anticipate their motion.

Correlation between the axis/plane of symmetry and direction of motion is one of the regularities in the animate world, which is not necessarily prescribed by inanimate Newtonian motion. The human visual system may have internalized such a regularity in the course of evolution (Shepard, 1984, 1987). There is some evidence that evolution has attuned biological visual systems to registering, tracking, and anticipating animate motion (Blake, 1993; Freyd \& Miller, 1992; Heptulla-Chatterjee, Freyd, \& Shiffrar, 1996; Miller \& Freyd, 1993).

If a symmetric object moves off the axis of symmetry, human observers may experience the axis and direction of motion to be more aligned than it actually is, and in so doing misperceive direction of motion. If such a tendency or bias exists, it could have significant consequences in the real world today. Quickly predicting the trajectory of man-made vehicles such as aircraft, spacecraft, and motor vehicles is vital in avoiding collisions or shooting targets. Yet these high-speed modern objects do not necessarily move along their axis of symmetry. For example, automobiles may skid, aircraft typically glide off their axes, and 
spacecraft in outer space can maneuver in any direction independent of hull orientation. Therefore, it is important to study whether there is such a perceptual bias.

Traditionally, perception of form and perception of motion have been treated as separate issues except for a few studies. For example, importance of axes of symmetry in rotational motion has been shown by Farrell and Shepard (1981) and Shiffrar and Shepard (1991). Pani, William, and Shippey (1995) showed that alignment between the axis of rotation and the shape-defined axis of the rotating object greatly facilitates accurate perception of rotation. Effects of shape orientation on perceived curvature of apparent motion path were studied by $\mathrm{McBeath}$ (1997), McBeath and Shepard (1989), and Proffit, Gilden, Kaiser, and Whelan (1988). However, the influence of symmetry on perceived direction of smooth translational motion has not been studied.

Suppose a symmetric object is moving $45^{\circ}$ from vertical, but its axis of symmetry is tilted $25^{\circ}$ from vertical (i.e., $20^{\circ}$ from the direction of motion): Observers may perceive the motion direction to be somewhere in between those two orientations (Figure 1). Experiment 1 was conducted to test this hypothesis.

\section{EXPERIMENT 1}

\section{Method}

Subjects. Ten undergraduates ( 8 females and 2 males) participated as subjects in partial fulfillment of an introductory psychology course requirement. All had normal or corrected-to-normal vision and were naive as to the purpose of the experiment. Throughout this study (Experiments $1-5$ ), none of the subjects participated in more than one experiment.

Apparatus and Stimuli. Stimuli were presented on a computer screen controlled by a Silicon Graphics IRIS 2400 workstation. Stimulus patterns were 18 -sided polygons, three asymmetric and three symmetric (see the two left columns of Figure 2). The coordinates of 18 vertices of each asymmetric polygon were chosen at random with certain constraints. The three asymmetric polygons in the leftmost column were originally devised by Farrell and Shepard (1981). The three symmetric polygons were made by patching together the

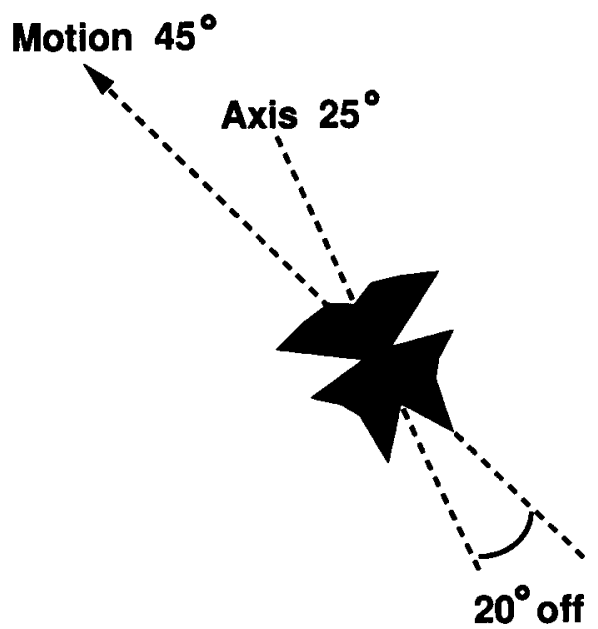

Figure 1. An example of a symmetric object moving off the axis of symmetry. left half of each asymmetric polygon with its mirror image. Though the asymmetric shapes did not have any intrinsic axes, the vertical line that went through the center of the shapes was defined as a control "axis." Thus, the left halves of the corresponding asymmetric and symmetric shapes were identical. Each polygon subtended about $3.3^{\circ}$ of visual angle in diameter on the display. The polygons were black on a white background to eliminate a luminous trail behind the moving shapes due to slow decay of the phosphor.

Design. Four directions of motion were used: $\pm 30^{\circ}$ and $\pm 45^{\circ}$ from vertical. Shapes were oriented $0^{\circ}, \pm 10^{\circ}, \pm 20^{\circ}$, and $\pm 30^{\circ}$ relative to each direction of motion. Each of the six shapes was presented once in combination with each of the four directions of motion and each of the seven axis orientations, totaling 168 trials per subject. Order of trials was randomized.

Procedure. The viewing distance was approximately $60 \mathrm{~cm}$. When a subject pressed the start key to begin each trial, a shape appeared a little below the center of the display (Figure 3A). The shape immediately started moving obliquely upward in one of the four directions at the speed of $3.9^{\circ} \mathrm{arc} / \mathrm{sec}$. The motion spanned $11^{\circ}$ arc in $2.8 \mathrm{sec}$. An arrow, $5.5^{\circ}$ arc long and $10^{\circ}$ arc below the motion path, was also presented. At the beginning of each trial, the arrow was vertical. It rotated clockwise or counterclockwise as a subject pressed the response keys. Subjects were instructed to adjust orientation of the arrow so that it appeared parallel to the path along which the shape was moving. Because this response method was not very quick, the moving shape was presented twice along the same path in each trial in order to allow subjects sufficient time. There was a 400-msec blank interval between the end of the first path and the start of the second path in order to prevent backward apparent motion. To ensure that subjects paid attention to shapes, they were instructed to remember how many different shapes were presented regardless of orientation. Subjects were also instructed to pursue the shapes with their eyes and not to tilt their heads. Subjects were given 10 practice trials at the beginning. Room illumination was kept dim but not completely dark. Each session took about $30 \mathrm{~min}$.

\section{Results and Discussion}

Figure 4 shows the mean magnitude of angular bias as a function of the angular departure of the shape axis from the direction of motion. The data were collapsed over three shapes and four directions of motion. A repeated measures analysis of variance (ANOVA) was performed. The slope for the symmetric shapes was highly significant $[F(1,9)=39.78, p<.0001]$. The slope for the asymmetric shapes was not significantly different from zero. The interaction between the two slopes was highly significant $[F(1,9)=29.81, p<.0004]$.

Asymmetric shapes did not cause any systematic bias, whereas symmetric shapes caused systematic biases: Subjects set the arrow at an orientation between direction of motion and the axis of symmetry. In general, the larger the angular departure of symmetry axis from direction of motion, the larger the bias, except that the bias seemed to level off or even decrease when the axis was more than $20^{\circ}$ away from the $\pm 30^{\circ}$ moving directions. The largest mean bias obtained was $10.1^{\circ}$. These results support the hypothesis that people are biased to see symmetric objects move along their axes of symmetry.

\section{EXPERIMENT 2}

In Experiment 1, subjects were asked to set the arrow parallel to the motion path. This "parallel setting" task 


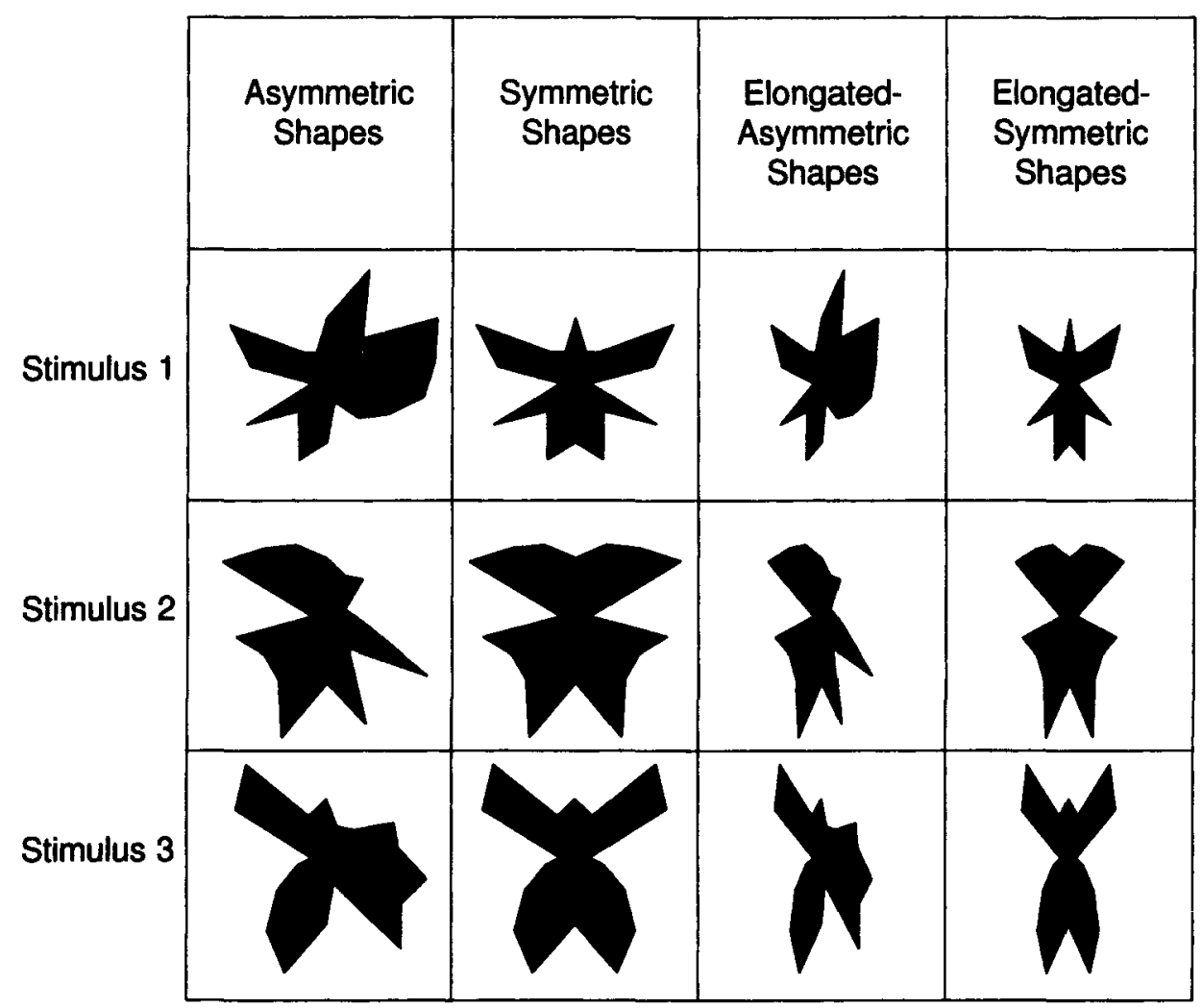

Figure 2. Random polygons used in the present study. The shapes in the left two columns were used in Experiments 1-4. The elongated versions (the right two columns) were used in Experiment 5. The shapes in the leftmost column were originally devised by Farrell and Shepard (1981).

was not very realistic. Besides, when trying to avoid collision, people usually would not have a second chance to see the path again. Experiment 2 was designed to see whether the bias would still arise in a more realistic task somewhat similar to catching. Because the task in Experiment 2 allowed a faster response than the task in Experiment 1 , the motion path was presented only once. In addition, in Experiment 1 the females seemed to exhibit a larger bias than the males, but there were not enough males (only 2 ) to test for a gender difference. Experiment 2 addressed the issue of a possible gender difference as well.

\section{Method}

Subjects. Eight male and 8 female undergraduates served as subjects. All were naive as to the purpose of the experiment and had normal or corrected-to-normal vision.

Procedure. Experiment 2 used the same stimuli, design, and procedure as Experiment 1 except as follows. As shown in Figure $3 \mathrm{~B}$, instead of the arrow, a pair of black dots placed on an invisible circular orbit was used to indicate perceived direction of motion. The space between the dots was constant and slightly larger than the largest width of the shapes. Subjects could move the dots on the circular orbit, using a mouse. The diameter of the orbit was $27^{\circ}$ arc. When subjects pressed the start key, one of the shapes appeared near the bottom of the display and started moving upward in an oblique direction. The dots were always at the top center at the beginning of each trial. The motion path spanned $11^{\circ}$ arc and was presented only once in each trial. The shapes moved at the speed of $3.9^{\circ} \mathrm{arc} / \mathrm{sec}$ and were shown for $2.8 \mathrm{sec}$, then disappeared $7^{\circ}$ arc short of the invisible orbit. The subjects' task was to position the dots so that if the shape continued moving in the same direction, it would pass between the dots.

\section{Results and Discussion}

Because the path of moving shapes always passed through the center of the circular orbit of the dots, the bias was defined as the angle formed by the path and the line connecting the center of orbit and the midpoint between the dots. Figure 5 shows the mean magnitude of angular bias of all subjects. There was no significant difference between the $30^{\circ}$ and the $45^{\circ}$ paths. The data were collapsed over four directions of motion and three shapes in each category. A repeated-measures ANOVA with a between-subjects factor (gender) and within-subjects factors (symmetry and axis orientation) was performed. The slope for the symmetric shapes was highly significant $[F(1,14)=138.96, p<.0001]$. The largest mean bias exhibited was about $-9^{\circ}$ at $-30^{\circ}$. The slope for the asymmetric shapes was not significantly different from zero $[F(1,14)=0.97, p<.34]$. The interaction between the two slopes was highly significant $[F(1,14)=111.9, p<.0001]$. 

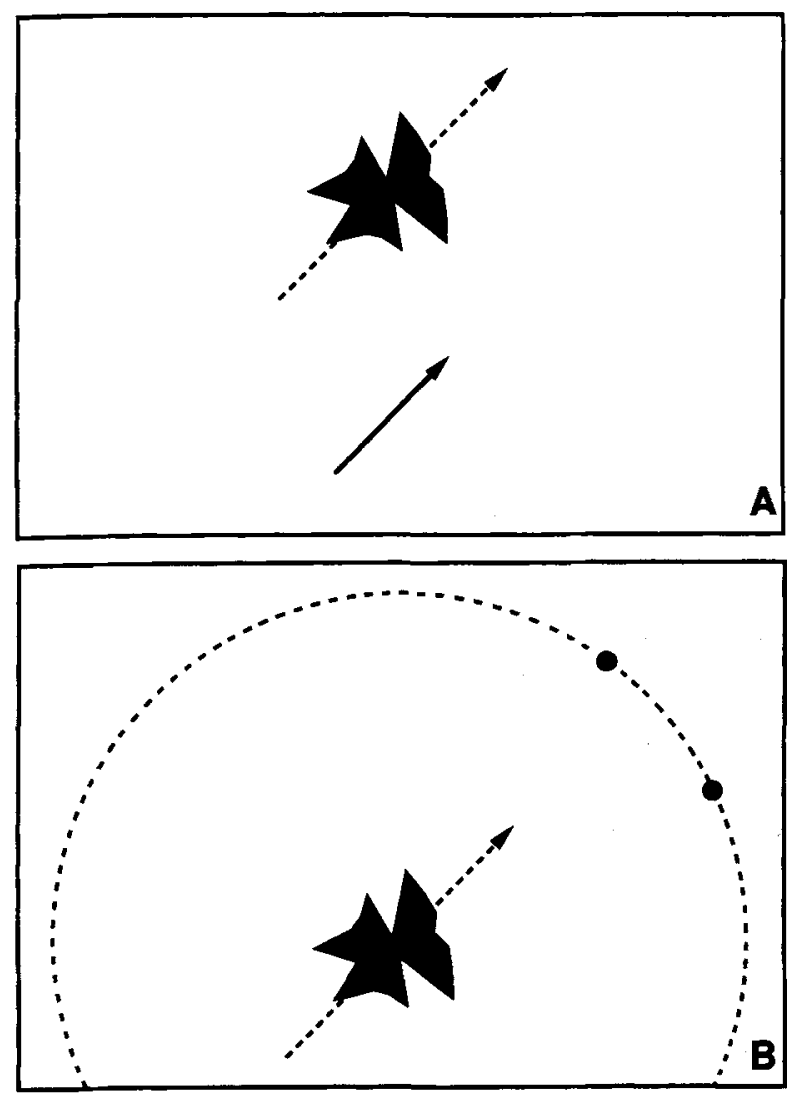

Figure 3. A: The procedure used in Experiments 1, 3, and 5. An obliquely moving shape was presented in the upper part of a computer screen. An arrow was presented below. Subjects adjusted orientation of the arrow until it appeared parallel to the motion path (Experiments 1 and 5) or to the shape axis (Experiment 3). B: The procedure used in Experiment 2. An obliquely moving shape was presented. The shape disappeared before it neared an invisible circular orbit. Subjects adjusted the position of a pair of dots with a constant separation on the orbit until the shape would appear to pass between the dots if it continued moving.

Figure 6 shows data from males and females separately. The three-way interaction between slope, shape category, and gender was significant $[F(1,14)=4.97, p<.043]$, indicating that females exhibited a larger bias for symmetric shapes than males.

There might be an alternative interpretation for the fact that asymmetric shapes caused no bias: Subjects may have perceived arbitrary axes of some kind for the asymmetric shapes, which may have caused shape-specific biases in perceived direction of motion. Averaging across all three asymmetric shapes may have masked such biases. To rule out this possibility, the data from each shape in Experiment 2 were analyzed separately. The linear regression slopes for the magnitude of bias as a function of relative shape orientation were $0.277,0.348$, and 0.337 for the symmetric shapes 1,2 , and 3 , respectively. All these slopes were statistically significant $(p<.001)$. The slopes for the asymmetric shapes 1,2 , and 3 were $-0.005,0.019$, and 0.013 , respectively; none were statis- tically significant. Even the smallest slope for a symmetric shape was 15 times as large as the steepest slope for an asymmetric shape. Evidently, subjects did not perceive any arbitrary axis for the asymmetric shapes that might have caused a bias.

Thus, Experiment 2 replicated the results of Experiment 1 in a more realistic task. As the angular departure of the symmetry axis from the direction of motion increased, the bias to see the motion toward the axis increased, whereas asymmetric shapes caused no bias that varied with their orientation. Experiment 2 also showed a significant gender difference. The origin of the gender difference is unknown, but it is consistent with past research on spatial judgments such as the rod-and-frame task, in which females are more influenced by context stimuli than are males (e.g., Harris, 1981; for a recent review, see Kimura \& Hampson, 1994).

Does the bias work the other way around? Does direction of motion influence perceived orientation of symmetric shapes? Bucher and Palmer (1985) showed that people tend to see a triangle pointing in the direction in which it is moving. It suggests that people might see symmetric shapes pointing (thus, oriented) more toward direction of motion. Furthermore, there is a possibility that subjects in Experiments 1 and 2 simply confused direction of motion and orientation of shapes, thus perceiving orientation of symmetric shapes seen to be more aligned with direction of motion than it actually was. Experiment 3 was conducted to answer these questions.

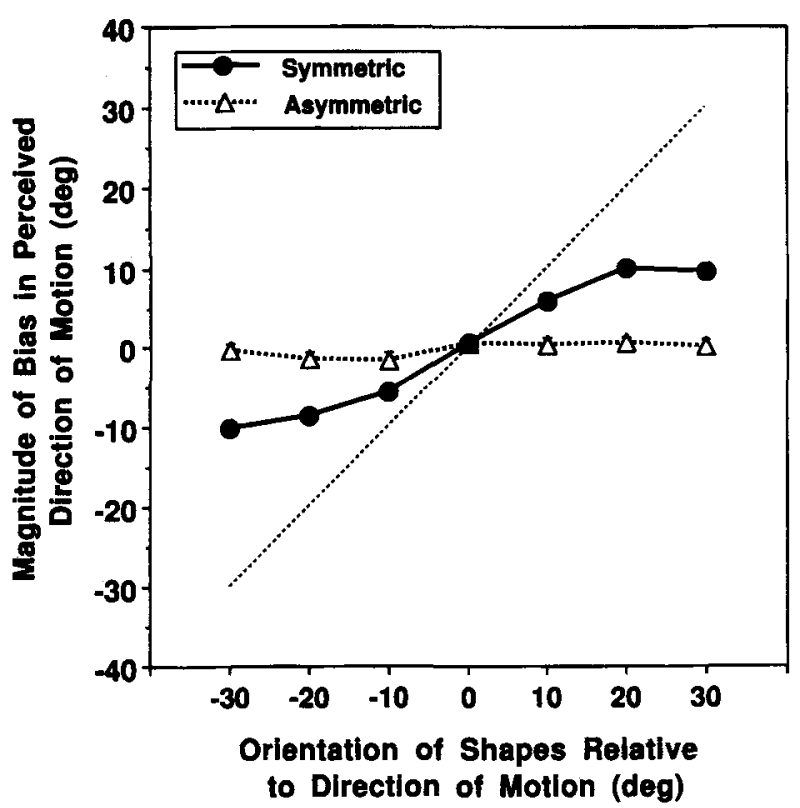

Figure 4. The results of Experiment 1. Mean angular bias in perceived direction of motion was plotted as a function of orientation of shapes relative to direction of motion. Counterclockwise was defined as positive. The diagonal broken line indicates the hypothetical magnitude of bias where the perceived direction of motion is completely aligned with the axis of symmetry. Error bars indicate the standard error of all pooled data. 


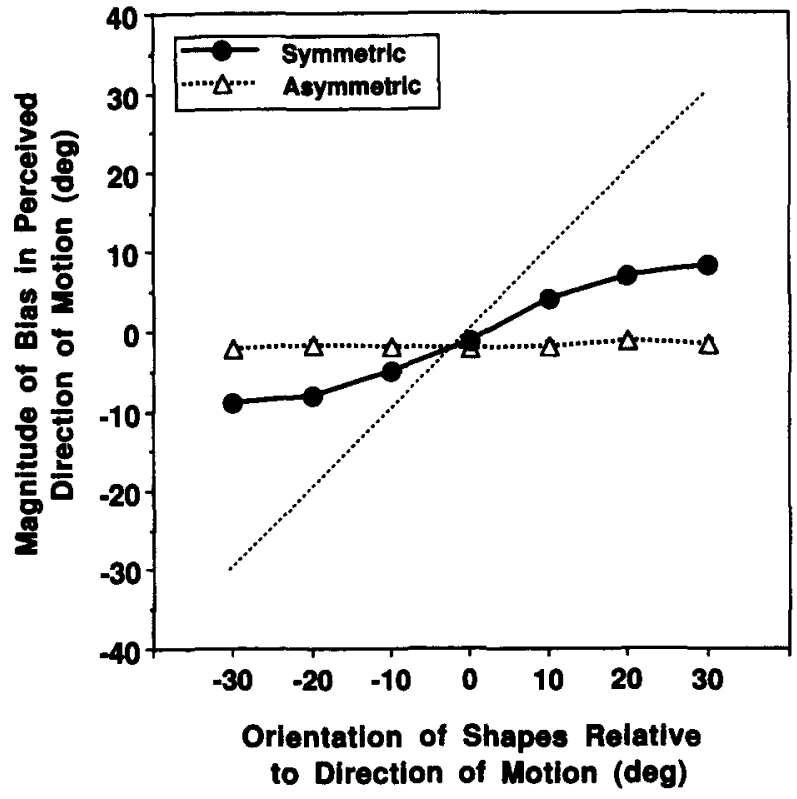

Figure 5. The results of Experiment 2. Mean angular bias in perceived direction of motion was plotted as a function of orientation of shapes relative to direction of motion. The diagonal broken line indicates the hypothetical magnitude of bias where the perceived direction of motion is completely aligned with the axis of symmetry. Error bars indicate the standard error of all pooled data.

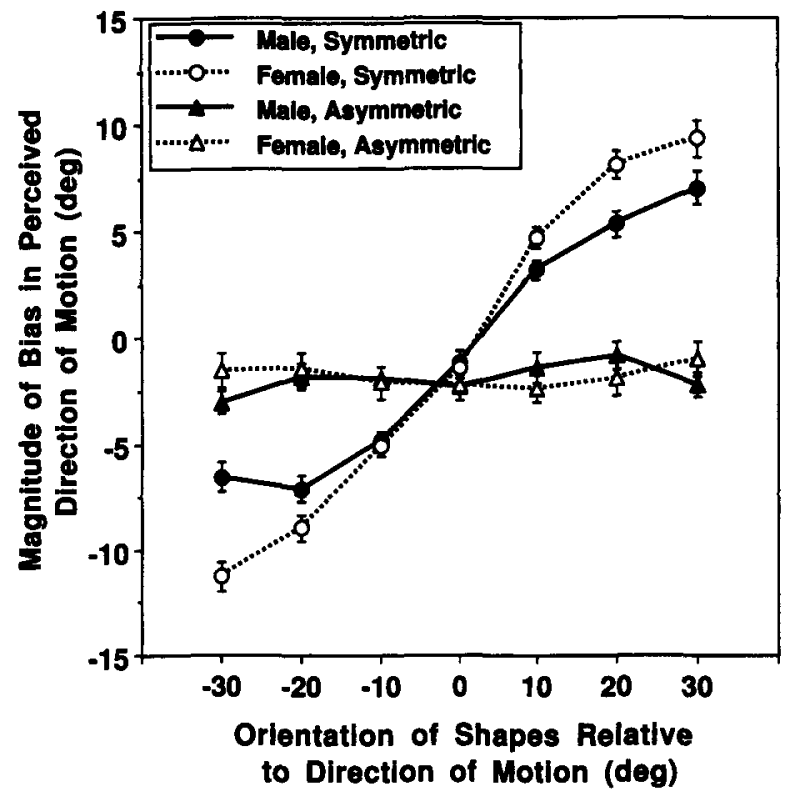

Figure 6. Angular biases for males and females in Experiment 2 were plotted separately. Error bars indicate the standard error of all pooled data.

\section{EXPERIMENT 3}

Method

Subjects. Eight female undergraduates served as subjects. All were naive as to the purpose of the experiment and had normal or corrected-to-normal vision.
Stimuli. The same three symmetric shapes and the same motion paths as in Experiments 1 and 2 were used. Asymmetric shapes were not presented. As in previous experiments, the three shapes were combined with the four directions of motion and seven axis orientations, totaling 84 trials per subject.

Procedure. The procedure was identical to that of Experiment 1 (Figure 3A) except that subjects were instructed to set the arrow parallel to the axis of symmetry of the shapes, not to direction of motion.

\section{Results and Discussion}

Figure 7 shows the mean magnitude of bias. Subjects exhibited a slight tendency to see orientation of symmetry axis toward direction of motion. But a repeated measures ANOVA showed that the slope was not significantly different from zero $[F(1,7)=3.46, p<.105]$. The largest mean bias exhibited was only $2.2^{\circ}$, which was much smaller than the largest mean effect (about $11^{\circ}$ ) of symmetric shape orientation on perceived direction of motion exhibited by female subjects in Experiments 1 and 2.

In Experiment 2, female subjects showed a greater effect of symmetry than male subjects. Because female subjects did not exhibit a significant trend in Experiment 3, male subjects would be even less likely to show an influence of motion on orientation. Direction of motion might slightly affect perceived orientation. But it can be concluded that effects of shape symmetry on perceived direction of motion are far greater than effects of direction of motion on perceived orientation of symmetric shapes. Thus, the results of Experiments 1 and 2 cannot be attributed to simple confusion between axis orientation and direction of motion.

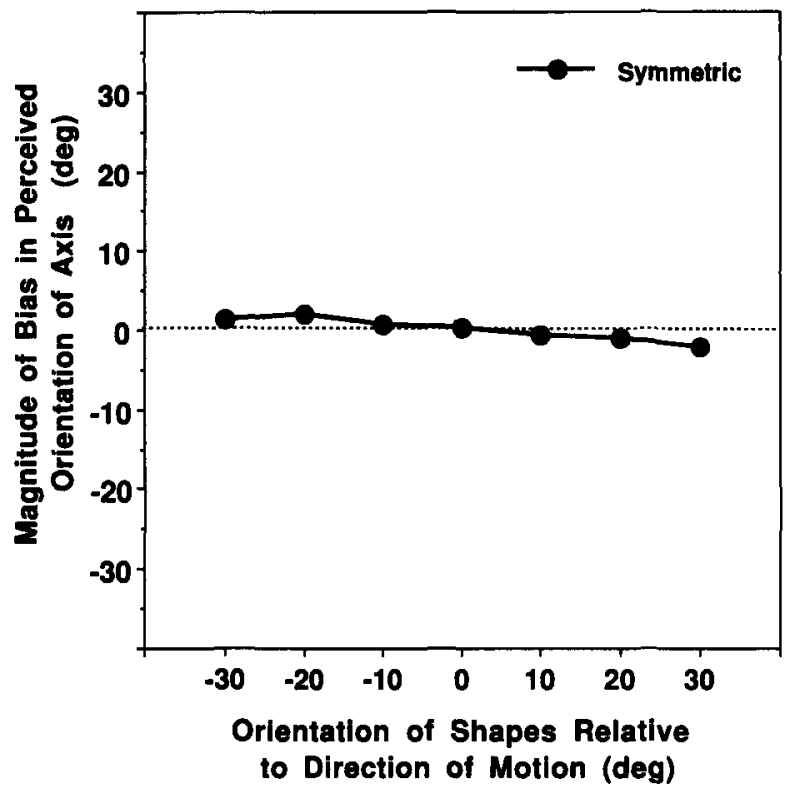

Figure 7. The results of Experiment 3. Mean angular bias in perceived orientation of symmetry axis was plotted as a function of angular difference between shape orientation and direction of motion. Counterclockwise was defined as positive. Error bars indicate the standard error of all pooled data. 


\section{EXPERIMENT 4}

Experiments 1 and 2 showed that observers have a bias to see symmetric shapes move along the axis of symmetry. However, it is possible that these results reflected some sort of "demand characteristics" that subjects guessed while performing the tasks. To rule out such an interpretation, Experiment 4 was designed to provide converging evidence in terms of reaction time (RT), using a facilitation/ interference paradigm. Symmetry of shapes may facilitate perceptual judgment on axis-aligned motion and/or interfere with judgment on off-axis motion.

\section{Method}

Subjects. Thirty-five undergraduates served as subjects. All were naive as to the purposes of the experiment and had normal or corrected-to-normal vision.

Stimuli. The same six shapes as in Experiments 1 and 2 were used. To present an "axis" explicitly, a white thin vertical line was added to the center of each symmetric and asymmetric shape in the two left columns of Figure 2. The white line was 1 pixel wide and 72 pixels $\left(2.0^{\circ}\right.$ arc $)$ long, splitting each shape at the center.

Procedure. Before beginning the experiment, subjects were informed that motion was equally likely to be axis aligned or off the axis regardless of shapes. When subjects pressed the start key, a shape with a white axis started moving in one of the four directions (i.e., $\pm 30^{\circ}$ or $\pm 45^{\circ}$ from vertical). On half the trials, the axis was aligned with direction of motion ( $0^{\circ}$ condition) and on the remaining trials oriented $\pm 20^{\circ}$ relative to direction of motion. Each motion path was presented only once in each trial, spanning $11^{\circ}$ arc and lasting $2.8 \mathrm{sec}$. Subjects judged whether a shape was moving along or off its axis as quickly as possible, then responded by pressing one of two keys. RT was measured. Each shape was combined with every direction and relative orientation in random order, totaling 96 trials per subject. Prior to the experiment, each subject was informed that motion would be aligned with the axis $50 \%$ of the time regardless of shapes. Subjects were given 10 practice trials.

\section{Results and Discussion}

Only the correct responses that were made before the shape disappeared (i.e., RTs of $2,900 \mathrm{msec}$ or shorter) were used for analysis. RTs were collapsed across shapes and directions of motion and averaged within each subject. Figure 8 shows mean RTs. A repeated measures ANOVA showed that the main effect of shape was significant $[F(1,34)=7.24, p<.011]$, but the main effect of relative axis orientation was not $[F(1,34)=0.439, p<$ $.5]$. The interaction between shape and axis orientation was significant $[F(1,34)=6.61, p<.015]$. Separate analyses of each factor showed that only the difference between the $0^{\circ}$ symmetric and the $0^{\circ}$ asymmetric shapes was significant $[F(1,34)=20.64, p<.001]$. Mean percentages correct were $95.8 \%$ and $84.1 \%$ for the $0^{\circ}$ and $20^{\circ}$ symmetric shapes, and $90.9 \%$ and $82.6 \%$ for the $0^{\circ}$ and $20^{\circ}$ asymmetric shapes, respectively. The $0^{\circ}$ symmetric shapes were the easiest in terms of both RTs and accuracy. Therefore, the effect was not due to a speed-accuracy tradeoff.

Judging whether an axis of an object was aligned with direction of its motion was faster only when the object was symmetric and moving along its axis. It demonstrated

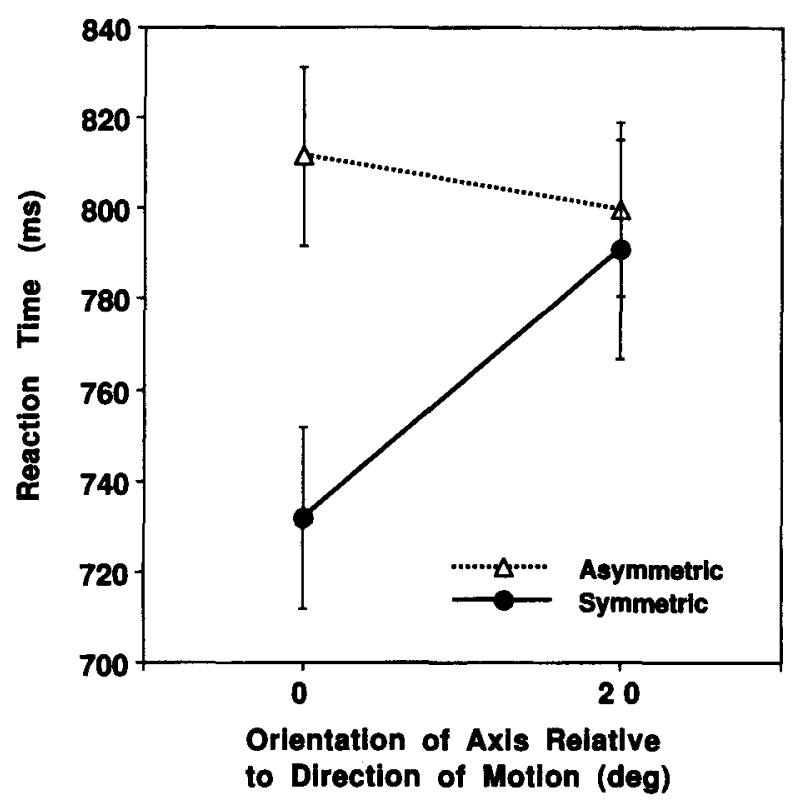

Figure 8. The results of Experiment 4. Reaction times for judging whether a shape was moving on or off its axis were plotted as a function of angular difference between axis orientation and motion direction. Error bars indicate the standard error of all pooled data.

that symmetry facilitates such a judgment when aligned with motion.

Symmetry is not the only regularity of animate motion. Because for most animals the primary direction of their locomotion is parallel to the long axes of their bodies, our visual system may be biased to see an elongated object move along the long axis. The purpose of Experiment 5 was to separate effects of elongation and symmetry on perceived direction of motion.

\section{EXPERIMENT 5}

\section{Method}

Subjects. Eight male and 8 female undergraduates served as subjects. All were naive as to the purposes of the experiment and had normal or corrected-to-normal vision.

Stimuli. The stimuli used are shown in the two right columns of Figure 2; they were made by halving the $x$-coordinates of the six shapes in the two left columns.

Procedure. The design and procedure were identical with those of Experiment 1 (Figure 3A). The procedure of Experiment 2 was not employed here because space between the dots necessary for the narrower shapes to pass through would have varied with orientation of the elongated shapes, which might have made the procedure somewhat inappropriate.

\section{Results and Discussion}

Figure 9 shows the mean bias of all subjects. A repeated measures ANOVA revealed that the overall slope for symmetric and asymmetric shapes was highly significant $[F(1,14)=47.04, p<.0001]$. The slope for the asymmetric shapes alone was highly significant $[F(1,14)=$ 


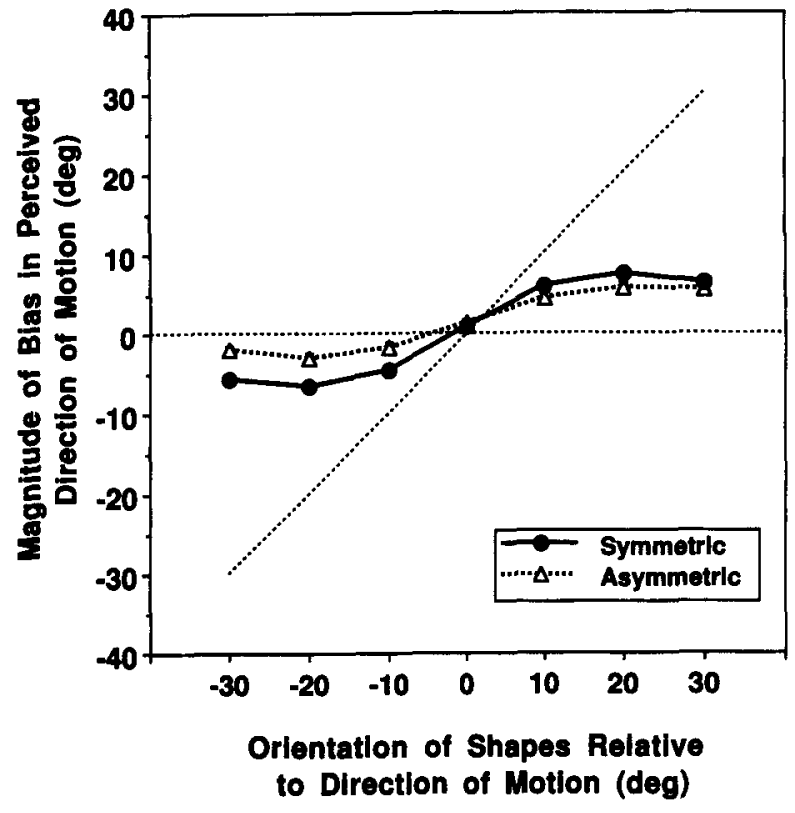

Figure 9. The results of Experiment 5. Mean angular bias in perceived direction of motion was plotted as a function of orientation of elongated shapes relative to direction of motion. Counterclockwise was defined as positive. The diagonal broken line indicates the hypothetical magnitude of bias where the perceived direction of motion is completely aligned with the axis of symmetry and/or elongation. Error bars indicate the standard error of all pooled data.

83.93, $p<.0001]$. The interaction between shape category and slope was also significant $[F(1,14)=11.61, p<.004]$, indicating that symmetric shapes caused a greater bias than asymmetric shapes. The interaction between gender and slope for the asymmetric shapes was significant $[F(1,14)=7.52, p<.016]$, indicating that females exhibited a larger effect of elongation than males. Three-way interaction among shape category, slope, and gender was marginal $[F(1,14)=3.68, p<.076]$.

The most significant findings of Experiment 5 were that even asymmetric shapes caused bias when they were elongated, and that symmetry caused bias above and beyond effects of elongation. Effects of elongation and symmetry seem to be additive to some extent. Overall bias for elongated symmetric shapes that 8 females exhibited in Experiment 5 was about the same size as bias for nonelongated symmetric shapes that 8 females exhibited in Experiment 1 (about $11^{\circ}$ maximum).

There are two explanations for why elongated and symmetric shapes in Experiment 5 did not cause a larger bias than nonelongated symmetric shapes in Experiment 1. First, it could be a ceiling effect: $A$ bias of about $11^{\circ}$ could be the maximum regardless of stimulus conditions. Second, the shapes used in Experiment 5 had only half the width (hence half the area) of those in Experiment 1 . The narrower width may have made the shape outlines less discernible. If symmetry became less salient, it might have attenuated the magnitude of bias. Further experiments are necessary to determine which explanation is true. Experiment 5 also replicated the gender difference found in Experiment 2. The gender difference existed not only for symmetry but also for elongation.

\section{GENERAL DISCUSSION}

Symmetry of an object biases perceived direction of its motion toward the axis of symmetry (Experiments 1 and 2 ). However, these interactions between shape and motion cannot be explained on the basis of a simple confusion between orientation of the axis of symmetry and orientation of motion path, because motion does not influence perceived orientation of the symmetry axis (Experiment 3 ). The bias shown in Experiments 1 and 2 is also evidenced by how fast subjects could make judgments about alignment between the axis of shape symmetry and direction of motion (Experiment 4). Orientation of elongated shapes also biases perceived direction of motion in much the same way as the bias caused by symmetry (Experiment 5 ).

These findings are consistent with the real-world regularity that the primary locomotion of most animals is in the direction of the axis/plane of bilateral symmetry and/ or the long axis of their bodies. However, the present experiments differ from the real world in one important aspect: Animals are 3-D objects moving in a 3-D world, often in such a way that neither their plane of bilateral symmetry nor their direction of 3-D motion is explicit in the retinal image. Thus, it remains to be seen whether observers exhibit a bias similar to the one reported in the present study for experiments that approximate typical 3-D situations.

These results may have been contingent on the procedure in which subjects were instructed to pursue the shapes with their eyes, which is what humans naturally do when seeing a moving object. This procedure was adopted in order to ensure that subjects would see details of the shapes using central vision. Had subjects seen the shapes only in the peripheral visual field, shapes would have appeared as blurry blobs, and the effect of symmetry and/ or elongation would probably have diminished.

In the present study, the direction of motion was always oblique; vertical or horizontal motion was not tested. This was because the discrimination threshold for orientation was smaller along vertical or horizontal than along oblique directions, and thus a perceptual bias would have been smaller for vertical or horizontal motion. The magnitude of misperception would have been maximal for oblique directions. This has been demonstrated for perception of rotation in 3-D space (Pani et al., 1995).

Perception of translational motion of symmetric/elongated objects exhibits characteristics similar to perception of rotational motion. Pani et al. (1995) found that when the axis of rotation of an object (a 3-D generalized cone) was not aligned with the object's generalized conic axis, observers often misperceived the orientation of the 
rotation axis in the 3-D space (see also Farrell \& Shepard, 1981; Shiffrar \& Shepard, 1991). Furthermore, Pani et al. found that perception of rotation was more accurate when the axis of elongation of an object was aligned with the rotation axis.

Although many studies have shown that reflectional (i.e., bilateral) symmetry can be more easily detected than other types of symmetry (for a review, see Wagemans, 1995), there are some reservations to the saliency of reflectional symmetry (Pomerantz \& Kubovy, 1986). Reflectional symmetry may not be as important in rotational motion as in translational motion. For example, reflectional and/or rotational symmetry of an object's crosssection about the axis of rotation does not facilitate accurate perception of rotation (Pani et al., 1995). Whether motion is translational or rotational, however, the human visual system seems to favor simpler spatiotemporal representations of motion (i.e., alignment among salient axes).

Werkhoven, Snippe, and Koenderink (1990) showed that perception of apparent motion is stronger between short lines oriented along the motion direction than between short lines oriented perpendicularly to it. Moreover, Post and Chaderjian (1987) showed that when a vertical or horizontal bar is moving obliquely, observers tend to overestimate the component of motion parallel to the long axis of the bar (see also Brown, 1931). Post and Chaderjian attributed this bias to elongation alone. But they confounded effects of elongation and symmetry, because the bar stimuli they used were both elongated and symmetric. The present study showed that elongation alone can influence perceived direction of motion in addition to the influence of symmetry.
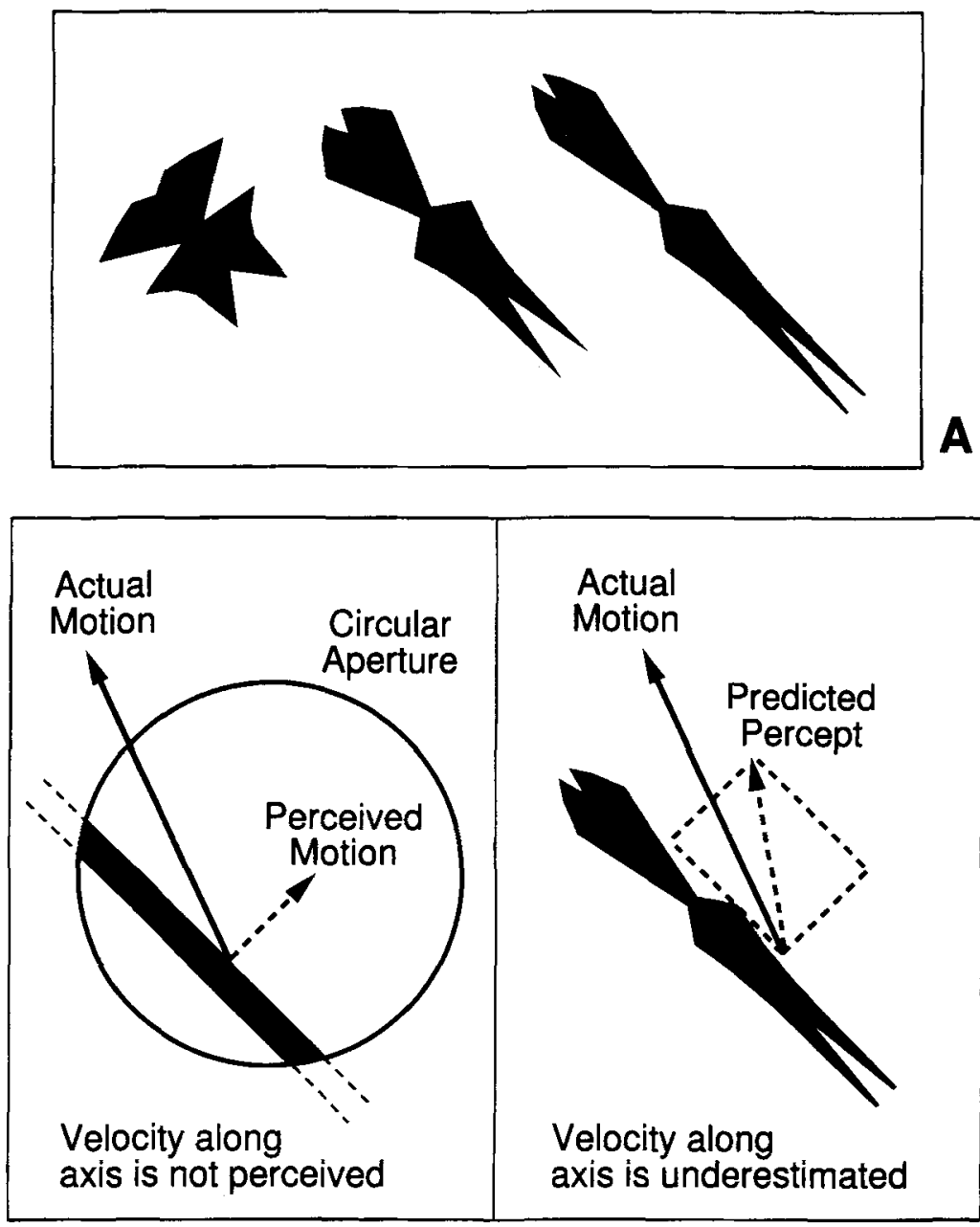

B

Figure 10. Relationship between elongation of shapes and the "aperture problem." A: Extreme elongation obscures features orthogonal to the long axis, making the shape look more like a bar. B: Motion of a bar seen through a circular aperture is perceived to be in the direction perpendicular to the bar. $C$ : The aperture problem would predict a motion bias perpendicular, not parallel, to the long axis, and thus could not explain the bias found in Experiment 5. 
Brown (1931) and Post and Chaderjian (1987) also showed that subjects overestimate the vertical component of motion along an oblique path. This finding cannot account for the present findings, however, because orientation of shapes used in the present study was counterbalanced relative to direction of motion.

One possibility, though, is that as a shape becomes longer, its asymmetric details become less salient and the overall envelope of the shape takes on a somewhat symmetric, bar-like appearance. Whether the effect of elongation on apparent direction of motion is mediated by approximate symmetry of the envelope is an open question.

Figure 10A illustrates that as a shape is elongated, components of its contour orthogonal to the axis of elongation become flattened and less salient. If it were elongated further, it would appear more like a bar or line, which would obscure the motion vector parallel to the long axis. Figure $10 \mathrm{~B}$ shows that motion of a bar whose ends are occluded behind a circular aperture is perceived to be in the direction orthogonal to the long axis of the bar, regardless of the actual direction of motion. This is because the velocity component parallel to the axis cannot be perceived. Likewise, Figure $10 \mathrm{C}$ shows that if the velocity component parallel to the axis of elongation were merely underestimated, perceived direction of motion would be biased away from the long axis (i.e., more perpendicular to the axis). However, what the present study showed was a bias toward the long axis (i.e., more parallel to the axis). Therefore, the prediction from the "aperture problem" cannot explain the present findings.

At what level of the visual system do the biases occur? There is some evidence that symmetry can be detected preattentively (e.g., Driver, Baylis, \& Rafal, 1992; Julesz, 1971; Locher \& Wagemans, 1993; Wolfe \& FriedmanHill, 1992). Symmetry detected in early vision may interact with motion processing. Werkhoven et al. (1990) suggested that the dominance of apparent motion parallel to the lines might be explained in terms of characteristics of spatiotemporal filtering in early vision. Bucher and Palmer (1985) speculated that interactions between the shape of a moving object and its perceived orientation might be mediated by low-level motion detectors. On the other hand, real-world regularities of animate motion may be represented cognitively at higher levels of visual processing, thus requiring attention. Whether the biases found in the present study occur at relatively early levels of the visual system or at higher cognitive levels should be resolved by further experiments.

Bucher and Palmer (1985) showed that perceived pointing of an equilateral triangle is affected by direction of its motion: When a triangle is moving along one of its three symmetry axes, observers are more likely to see the triangle point in that direction than when it is stationary or moving along a side of the triangle. In axis-aligned motion, motion vectors of all the points composing the triangle are also symmetric about the axis. Bucher and Palmer suggested that this symmetric nature of the "spacetime event" (i.e., motion vectors) causes the bias in per- ceived orientation. Experiments 1 and 2 in the present study demonstrated the reverse bias: Orientation of moving symmetric shapes biases perceived direction of motion toward the axis of symmetry.

When direction of motion of symmetric shapes is somewhat off the axis, the resulting space-time event is somewhat asymmetric. The bias to see the axis and motion more aligned than they actually are can be interpreted as the bias to perceive a somewhat asymmetric space-time event as more symmetric than it actually is. Freyd and Tversky (1984), Tversky and Schiano (1989). Schiano and Tversky (1992), and McBeath et al. (1997) showed that observers have a bias toward perceiving and remembering static, slightly asymmetric forms as more symmetric than they actually are. The present study demonstrated that such a bias also occurs in the space-time domain.

\section{REFERENCES}

BARLOW, H. B., \& ReEves, B. C. (1979). The versatility and absolute efficiency of detecting mirror symmetry in random dot displays. Vision Research, 19, 783-793.

Blake, R. (1993). Cats perceive biological motion. Psychological Science, 4, 54-57.

Brown, J. F. (1931). The visual perception of velocity. Psychologische Forschung, 14, 199-232.

BUCHER, N. M., \& PALMER, S. E. (1985). Effects of motion on perceived pointing of ambiguous triangles. Perception \& Psychophysics, 38, 227-236.

Carmody, D. P., Nodine, C. F., \& Locher, P. J. (1977). Global detection of symmetry. Perceptual \& Motor Skills, 45, 1267-1273.

Corballis, M. C. (1976). The psychology of left and right. Hillsdale, $\mathrm{NJ}$ : Erlbaum.

Driver, J., Baylis, G. C., \& Rafal, R. (1992). Preserved figureground segregation and symmetry perception in visual neglect. $\mathrm{Na}$ ture, 360, 73-75.

ENQUIST, M., \& ARAK, A. (1994). Symmetry, beauty and evolution. $\mathrm{Na}$ ture, 372, 169-172.

FarRell, J. E., \& ShEPARD, R. N. (1981). Shape, orientation, and apparent rotational motion. Journal of Experimental Psychology: Human Perception \& Performance, 7, 477-486.

FReyd, J. J., \& Miller, G. F. (1992, November). Creature motion. Paper presented at the annual meeting of the Psychonomic Society, St. Louis.

Freyd, J. J., \& TVERSKY, B. (1984). Force of symmetry in form perception. American Journal of Psychology, 97, 109-126.

HaRrIS, L. J. (1981). Sex related variations in spatial skills. In L. S. Liben, A. H. Patterson, \& N. Newcombe (Eds.), Spatial representation and behavior across the life span: Theory and application (pp. 83-125). New York: Academic Press.

Heptulla-Chatterjee, S., Freyd, J. J., \& Shiffrar, M. (1996). Configural processing in the perception of apparent biological motion. Journal of Experimental Psychology: Human Perception \& Performance, 22, 916-929.

HochberG, J. E. (1978). Perception (2nd ed.). Englewood Cliffs, NJ: Prentice-Hall.

JulEsz, B. (1971). Foundations of cyclopean perception. Chicago: University of Chicago Press.

KimuRA, D., \& Hampson, E. (1994). Cognitive pattern in men and women is influenced by fluctuations in sex hormones. Current Directions in Psychological Science, 3, 57-61.

LOCHER, P., \& WAGEMANS, J. (1993). The effects of element type and spatial grouping on symmetry detection. Perception, 22, 565-587.

MARR, D. (1982). Vision. San Francisco: W. H. Freeman.

MCBEATH, M. K. (1997). The influence of object shape on the path of apparent motion. Manuscript submitted for publication.

McBeath, M. K., Schiano, D. J., \& TVersky, B. (1997). Three- 
dimensional bilateral symmetry bias in judgments of figural identity and orientation. Psychological Science, 8, 217-223.

MCBeath, M. K., \& ShePard, R. N. (1989). Apparent motion between shapes differing in location and orientation: A window technique for estimating path curvature. Perception \& Psychophysics, 46, 333-337.

MiLleR, G. F., \& FREYD, J. J. (1993). Dynamic mental representations of animate motion: The interplay among evolutionary, cognitive and behavioral dynamics (Tech. Rep. CSRP 290, ISSN 1350-3162). Brighton, U.K.: University of Sussex.

MøLLER, A.P. (1992). Female swallow preference for symmetrical male sexual ornaments. Nature, 357, 238-240.

PANI, J. R. (in press). Descriptions of orientation and structure in perception and physical reasoning. In E. Winograd, R. Fivush, \& W. Hirst (Eds.), Ecological approaches to cognition: Essays in honor of Ulric Neisser (pp. 59-95). Hillsdale, NJ: Erlbaum.

PaNi, J. R., William, C. T., \& ShiPPEy, G. T. (1995). Determinants of the perception of rotational motion: Orientation of the motion to the object and to the environment. Journal of Experimental Psychology: Human Perception \& Performance, 21, 1441-1456.

Pomerantz, J. R., \& Kubovy, M. (1986). Theoretical approaches to perceptual organization. In K. R. Boff, L. Kaufman, \& J. P. Thomas (Eds.), Handbook of perception and human performance: Vol. II. Cognitive processes and performance (pp. 36. 1-36.46). New York: Wiley.

Post, R. B., \& ChaderJian, A. M. (1987). Perceived path of oblique motion: Horizontal-vertical and stimulus-orientation effects. Perception, 16, 23-28.

Proffit, D. R., Gilden, D. L., Kaiser, M. K., \& Whelan, S. M. (1988). The effect of configural orientation on perceived trajectory in apparent motion. Perception \& Psychophysics, 43, 465-474.

Rock, I. (1973). Orientation and form. New York: Academic Press. SCHIANO, D. J., \& TVERSKY, B. (1992). Structure and strategy in encoding simplified graphs. Memory \& Cognition, 20, 12-20.
SHEPARD, R. N. (1984). Ecological constraints on internal representation: Resonant kinematics of perceiving, imagining, thinking, and dreaming. Psychological Review, 91, 417-447.

SHEPARD, R. N. (1987). Evolution of a mesh between principles of the mind and regularities of the world. In J. Dupre (Ed.), The latest on the best: Essays on evolution and optimality (pp. 251-275). Cambridge, MA: MIT Press.

Shiffrar, M. M., \& Shepard, R. N. (1991). Comparison of cube rotations around axes inclined relative to the environment or to the cube. Journal of Experimental Psychology: Human Perception \& Performance, 17, 44-54.

Swaddle, J. P., \& Cuthill, I. C. (1994), Preference for symmetric males by female zebra finches. Nature, 367, 165-166.

TVERSKY, B., \& SChiano, D. J. (1989). Perceptual and conceptual factors in distortions in memory for graphs and maps. Journal of Experimental Psychology: General, 118, 387-398.

WAGEMANS, J. (1995). Detection of visual symmetries. Spatial Vision, 9, 9-32.

Werkhoven, P., SNIPPE, H. P., \& Koenderink, J. J. (1990). Effects of element orientation on apparent motion perception. Perception \& Psychophysics, 47, 509-525.

WERTHEIMER, M. (1958). Principles of perceptual organization. In D. C. Beardslee \& M. Wertheimer (Eds.), Readings in perception (pp. 115135). Princeton, NJ: Van Nostrand.

Wolfe, J. M., \& Friedman-Hill, S. R. (1992). On the role of symmetry in visual search. Psychological Science, 3, 194-198.

(Manuscript received April 11, 1997; revision accepted for publication December 8,1997 .) 